\title{
Spiritualità, migrazione e psichiatria
}

\section{Un argomento scottante per la psicoterapia?}

\author{
Michele Mattia \\ Psychotherapie-Wissenschaft 9 (1) 77-82 2019 \\ www.psychotherapie-wissenschaft.info \\ CC BY-NC-ND \\ https://doi.org/10.30820/1664-9583-2019-1-77
}

\begin{abstract}
Riassunto: Sebbene la pratica psichiatrica e psicologica sia esercitata da secoli, le problematiche religiose e spirituali hanno continuato ad avere un impatto sulla prospettiva dei pazienti, in relazione alla loro salute e alla gestione dei disturbi psichici da cui sono afflitti. Le credenze religiose e spirituali sono prevalenti, più di quanto si possa pensare, fra tutti coloro che hanno un disturbo affettivo, ansioso o psicotico. Gli psicoterapeuti e gli psichiatri sono raramente consapevoli e consci dell'importanza che la religione può avere nel costrutto psichico del soggetto. Inoltre hanno difficoltà a comprendere i valori della spiritualità e della religione nella strutturazione dell'apparato psichico delle persone e di quanto questi valori possano sostenere o interferire nelle relazioni con la famiglia, la società, il lavoro, o con la costruzione della propria identità personologica. In questo articolo si presenteranno alcuni casi clinici per discutere la relazione fra religione, spiritualità e disturbi psichiatrici, particolarmente si metteranno in evidenza gli effetti pato-facilitatori, pato-induttori o pato-protettivi delle diverse pratiche religiose. Particolarmente sottolineremo la relazione fra le tre religioni monoteistiche (Cristiana, Islamica e Ebraica) e le altre tre fedi religiose quali il Buddismo, l'Induismo e la chiesa Evangelica. Verranno presentati sei casi clinici di cui uno su depressione ed ansia ed effetti protettivi di alcune pratiche dell'Induismo. Altri due casi verteranno sulla sintomatologia depressiva e gli effetti protettivi della religione cattolica. Un altro caso sarà sul cambiamento di credenza religiosa in un giovane studente svizzero di filosofia. Era cattolico e cambiò la sua religione diventando musulmano. Questa conversione creò inizialmente uno stato di importante riduzione dei disturbi psichiatrici (era un soggetto con una gravissima nevrosi), ma successivamente virò in uno stato aggressivo e violento. La sua mente non riusciva a rimanere calma e tranquilla, sviluppando un forte rischio di radicalizzazione. Un altro caso considererà gli effetti analgesici delle pratiche meditative del Buddismo. Un giovane uomo svizzero che aveva da molti anni differenti forme di dolore, dopo aver incontrato la religione buddista sviluppò la capacità di gestire il suo dolore riducendo in modo significativo l'uso degli analgesici e degli antidepressivi. L'ultimo caso esaminerà l'effetto rilassante pato-protettivo della chiesa evangelica in una donna con disturbo da attacco di panico e un bolus isterico. Si analizzeranno anche i pregiudizi che possono bloccare lo psicoterapeuta nella sua pratica clinica e i pattern da affrontare per evolversi dai propri blocchi interiori.
\end{abstract}

Parole chiave: Psichiatria culturale, Spiritualità, Religione, Credenze, Coping, Pregiudizi, Psicoterapia

\section{Introduzione}

«Dio, gli Dei, il potere divino o gli Spiriti esistono in tutte le culture e in tutto il mondo.»

Koenig et al. (2001)

La religiosità e le credenze hanno un impatto significativo sulle persone, sulla loro salute mentale e somatica e sulla gestione dei disturbi di cui sono afflitti. Le credenze religiose, in accordo con la maggior parte delle ricerche, sono prevalenti fra coloro che hanno un disturbo delle emozioni o una malattia mentale.

I clinici sono raramente consapevoli dell'importanza delle credenze religiose e comprenderle darebbe una forza terapeutica maggiore al trattamento (Koenig et al., 2005). Le comunità scientifiche e teologiche sono divise su come definire i concetti di religione e spiritualità. Il concetto di religione è adoperato per indicare dei comportamenti specifici, sociali, dottrinali e per definire delle caratteristiche dei gruppi che appartengono alle diverse aree religiose.

In particolare il termine religione coinvolge le credenze in un potere sovrannaturale o in un essere trascendente, nella verità o nella realtà massima, e l'espressione di tali credenze risiede nei comportamenti e nei rituali.

La religione è un sistema organizzato di credenze, pratiche e rituali orientate al sacro. La spiritualità è un termine che concerne la questione massima nei confronti del significato della vita e tutto ciò che è relativo al trascendente, che può o potrebbe anche non sorgere dalle tradizionali forme religiose (ma usualmente deriva da queste).

La spiritualità concerne esperienze personali o ricerche nei confronti del trascendente non necessariamente collegate ad una determinata religione, che va oltre la semplice affiliazione ad una religione. Alcuni sottolineano che la definizione di spiritualità è più soggettiva, meno misurabile. In una prospettiva clinica avere un termine che è così ampio e diffuso è positivo poiché ciò permette ad ognuno di definire il proprio significato, mantenendo la soggettività. Il linguaggio della spiritualità aiuta a 
stabilire un dialogo con le persone religiose o anche non religiose (Larson et al., 1997).

\section{Il Ruolo del Terapeuta}

Il ruolo del clinico non è ovvio nei confronti di queste situazioni. Gli psicoterapeuti suppongono di avere molteplici ragioni per illustrare la loro ritrosia nei confronti delle questioni spirituali e religiose nelle terapie.

In primis gli psicoterapeuti hanno un loro proprio coinvolgimento religioso - o uno scarso coinvolgimento religioso - che può influenzare il loro interesse nelle questioni religiose o spirituali. In generale gli psicoterapeuti sono meno coinvolti nelle attività religiose che gli stessi pazienti e ciò comporta che sono meno interessati a discutere di queste problematiche in terapia (Neeleman $\&$ King, 1993).

In seconda istanza gli psicoterapeuti hanno una scarsa conoscenza di come intraprendere il discorso religioso e spirituale nel setting clinico. Durante gli studi universitari o post-universitari raramente vi sono delle formazioni specifiche concernenti la questione religiosa o spirituale. Non bisogna dimenticare, poi, che vi sono sempre stati, storicamente, conflitti significativi fra la psichiatria, la psicologia e la religione.

Freud ha descritto la religione come una «nevrosi ossessiva dell'umanità», ovvero una difesa nevrotica contro le vicissitudini dell'esistenza, ed era convinto che le persone sarebbero state meglio senza il credo religioso (Gay, 1987). Questa immagine svantaggiosa è stata spesso enfatizzata dai professionisti della salute mentale, sminuendo i benefici della religione.

Rimane tuttora aperto un antagonismo fra gli psicoterapeuti e il clero, poiché i campi di interesse, in qualche modo, si sovrappongono e spesso si condividono gli stessi «persone/clienti/pazienti (?)».

Oltre ciò è noto come alcuni psicoterapeuti hanno il timore che parlare di questioni pertinenti alla religione potrebbe rappresentare un cammino in territori sconosciuti, rischiando così di creare danno al paziente stesso. Gli psicoterapeuti potrebbero sentire un senso di disagio nell'essere coinvolti in una rete di cura sociale dove i ruoli non sono ben definiti fra i clinici e i religiosi.

Da considerare anche altri fattori di resistenza nella comunicazione psicoterapeuta-soggetto nell'affrontare la questione religiosa o spirituale, che sono la relazione complessa fra medicina e religione; il tema troppo privato: il terapeuta che desidera imporre la propria religione o che non è esperto nel rapporto religione-salute ed infine i pazienti che non vogliono parlarne (Neeleman \& King, 1993). Un fattore comune di tutti questi elementi concerne la scarsa conoscenza del dominio della religione e della spiritualità e la presenza di pochi strumenti a disposizione.

Su scala globale con i soggetti che soffrono di disturbi della sfera psichica, appare cruciale comprendere la loro sofferenza sia in termini scientifici che spirituali, così da massimizzare la loro aderenza al trattamento, in modo da rinforzare la relazione terapeutica.

\section{Rapporto fra Religione, Medicina e Psichiatria}

Il processo della valutazione psichiatrica deve essere sensibile all'etnicità del soggetto, al suo luogo di nascita, al suo genere, alla classe sociale, all'orientamento sessuale e al credo religioso e spirituale (American Psychiatric Association, 1995).

Le ricerche suggeriscono che la religione e o la spiritualità possano essere di aiuto per le persone con disturbi somatici. Ad esempio i soggetti che appartengono a gruppi religiosi sono maggiormente protetti da disturbi cardiaci (Goldbourt et al., 1993). Questo potrebbe essere dovuto alla relazione fra le credenze religiose e i fattori di rischio cardiovascolari quale pressione arteriosa elevata, fumo, cattive abitudini alimentari, che sono ridotte nei loro effetti stressanti dal comportamento religioso.

La religione potrebbe anche influenzare l'incidenza tumorale particolarmente attraverso le buone abitudini alimentari e le pratiche salutari che sono presenti in alcuni gruppi religiosi (Enstrom, 1989). Il corso e i risultati dei tumori potrebbero anche essere favorevolmente influenzati dal coinvolgimento religioso attraverso un miglioramento dei comportamenti salutari, ma anche attraverso l'uso di un coping religioso che può aumentare la speranza e ridurre l'ansia.

Nel campo della psichiatria, particolarmente per ragioni storiche, l'attitudine generale verso la religione è stata sempre ambivalente. Le credenze religiose, le pratiche e le esperienze dirette sono spesso state considerate come una forma nevrotica dai professionisti della salute mentale, almeno del passato. La religione offre una via differente per osservare la malattia psichiatrica che può entrare in conflitto con gli psichiatri e gli psicoterapeuti stessi.

Il coping religioso è stato mostrato che influenza i risultati dell'elaborazione del lutto e del disturbo depressivo maggiore (Mohr \& Huguelet, 2004). Le ricerche effettuate in Svizzera, da alcuni autori e anche in altre nazioni, hanno documentato il positivo beneficio in termine di coping che la religione e/o la spiritualità può avere per i pazienti psicotici (Mohr \& Huguelet, 2004; Mohr et al., 2006; Yangarber-Hicks, 2004).

Gli psicoterapeuti dovrebbero considerare questa questione e discutere sempre con i loro pazienti delle loro pratiche religiose. È importante conoscere non unicamente se sono dei credenti, ma anche l'intensità dei loro sentimenti religiosi e la pratica spirituale e religiosa. Colucci e Martin (Colucci \& Martin, 2008) hanno descritto il più importante modello delle ricerche empiriche fra religione e suicidio.

Gli autori concludono che i fattori religiosi sono generalmente associati con una più bassa ideazione suicidaria, un'attitudine più negativa verso il suicidio e tassi di tentativi suicidari più bassi. In breve, la religione sembra che provveda ad una protezione maggiore contro i pensieri suicidari e i comportamenti suicidari, attraverso una migliore integrazione sociale (ad esempio l'appartenenza a gruppi religiosi), i contenuti delle credenze religiose, o l'aderenza alle norme sociali che non supportano l'ideazione suicidaria (Colucci \& Martin, 2008). 


\section{Basi Neurobiologiche Della Religione e Della Spiritualità}

I risultati che provengono dalle metanalisi suggeriscono che un alto grado di spiritualità è collegato con l'attivazione dei sistemi serotoninergici e dopaminergici. Il lobo frontale, la corteccia prefrontale e le connessioni col talamo, il lobo parietale posteriore e superiore e il sistema limbico, sono le rigioni più coinvolte (Newberg et al., 2001, 2003; Newberg \& Iversen, 2003; Muramoto, 2004).

Alcuni studi sull'attivazione delle vie serotoninergiche e dopaminergiche suggeriscono che livelli più elevati di attività nei due sistemi sono presenti non unicamente nei soggetti con un alto senso della spiritualità ma anche durante le attività religiose, quali la meditazione e la contemplazione (Cloninger et al., 1993, 1998).

La Speranza, l'Ottimismo, l'Amore e la Soddisfazione stimolano la produzione delle endorfine, inducendo un'attivazione del circuito del piacere e di conseguenza prevenendo i disturbi della sfera psichiatrica. Attraverso l'attivazione del sistema limbico e dell'asse ipofisario-ipotalamico-surrenalico rinforzano il sistema immunitario e diminuiscono il turnover degli ormoni dello stress riducendo, fra l'altro, la frequenza cardiaca, la pressione arteriosa, l'ansia e la depressione.

La spiritualità e la religione, attraverso i meccanisi neurobiologici descritti, possono avere un effetto tampone sugli stressors della vita creando una fonte di serenità interna e un senso di appagamento e di protezione dagli effetti patologici.

\section{Casi Clinici}

Il primo caso clinico presentato concerne una signora di 66 anni, cattolica. Proviene da modelli gentoriali cattolici praticanti, rigororosi. La famiglia al completo si recava tutte le domeniche a messa e partecipava attivamente alle funzioni e alle attività della parrocchia. Maestra delle elementari di professione. Sposata e madre di 3 figli adottati. Era arrivata in terapia per una problematica relazionale con il figlio, allora dedito all'uso della marijuana. Inoltre presentava un quadro depressivo.

Diagnosi di carcinoma mammario nel 2005, vescicale e ovarico nel 2008. Ogni volta seguendo le cure oncologiche specifiche, di concerto con un'aderenza alle preghiere e alla contemplazione ha superato le malattie. "... Se devo scegliere tra il cancro ed il figlio, scelgo il cancro ... il cancro è una passeggiata per me ...». Così esprimeva la sua angoscia verso il proprio figlio. Spesso presentava crisi d'ansia molto profonde, con inquietudine, tensione, insonnia, in una personalità con tratti intrusivi e ipercontrollanti.

Nel percorso di psicoterapia sistemica individuale, col supporto di una farmacoterapia specifica, abbiamo incluso l'area religiosa, particolarmente abbondante nella sua storia personale e nella sua quotidianità. È stata rinforzata la partecipazione alle preghiere mattutine e alla messa serale.
I fattori psicoscociali pato-protettivi dell'aderenza alla religione che sono emersi sono stati: appartenenza al gruppo, socializzazione, contemplazione nelle preghiere con conseguente riduzione dei sintomi e capacità di individuare aree di conforto soggettivo. Si è assisitito anche ad una riduzione degli stati di rabbia e dell'impulsività, nonché delle rimuginazioni a stampo ossessivo (particolarmente sul figlio).

Il secondo caso clinico si sofferma sulla storia di un uomo di 28 anni. "Il cordone si era attaccato al mio collo come un cappio ... mio padre, un uomo con un pessimo carattere, egoista, era violento con mia madre e con me ...». A causa dei maltrattamenti subiti, in età adulta ha chiesto ed ottenuto il cambio del cognome che lo identificava col padre. Dalla separazione genitoriale ha sempre vissuto con la madre. Ha un fratello maggiore ed uno minore, coi quali la relazione è disfunzionale.

È stato vittima di bullismo nelle medie, nonché deriso per il suo sovrappeso. Ha terminato la facoltà di filosofia, presso l'USI di Lugano.

Nella sua storia personale emerge l'isolamento relazionale come tratto specifico evolutivo ed attuale. Dal lato diagnostico siamo nell'area del disturbo di personalità narcisitica con tratti ossessivi marcati assieme all'area depressiva.

Dopo aver vissuto una grave frustrazione relazionale venendo ingiustamente accusato di avere rubato degli oggetti da un gruppo di amici, che egli aveva eletto come succedaneo della sua famiglia disgregata, ne venne espulso.

Da lì è sprofondato in uno stato di rabbia con spinta pulsionale reattiva ad entrare nella religione musulmana. Prima aveva cognizione di essere cattolico, ma non aveva nessun rapporto nè spirituale nè sociale con le pratiche religiose cattoliche o cristiane.

Dopo poche settimane dalla conversione all'islamismo si presentò coi capelli rasati e la barba lunga. "Sono diventato musulmano ... voglio imparare il corano a memoria, in arabo ...». Dopo qualche settimana affermò: «Ho bruciato tutti i farmaci perché non voglio più essere narcotizzato ...». Venne indottrinato dall'Islam più fuorviato nell'area prevaricatrice, integralista con derive «estremistiche» contro la civiltà occidentale e l'imperialismo americano.

Dopo qualche mese venne ricoverato presso la Clinica Psichiatrica Cantonale di Mendrisio, poiché era diventato molto aggressivo, anche fisicamente, contro la madre. La madre non tollerava che egli voleva imporle la religione islamica integralista.

Dopo questa crisi si allontanò dall'islamismo massimalista e aggressivo. Un anno dopo andò incontro ad un nuovo riavvicinamento all'Islam

«... quando l'estate scorsa sono entrato nell'Islam ero rimasto in uno stato di fascinazione dell'Islam ... adesso ho capito di non essere d'accordo con le loro verità fondamentali, anche se come religione monoteistica è l'unica in cui credo ... è ritornato in modo attivo il pensiero della religione, a livello di cervello, di mente ... si dimostrano con chiarezza le verità dell'islamismo... 
mi manca la preghiera ... vado in crisi per tutto e per essermi allontanato dall'Islam ... ho un senso di colpa e di dolore e di grande spaesamento...mi mancano punti di riferimento ... l'Islam diventa il mio punto di riferimento ...».

Frequenta regolarmente la Moschea. Il sabato sera si incontra con un gruppo di ragazzi, dai diciotto ai trent'anni, per studiare l'Islam, pregare e cenare assieme. La domenica va in Moschea con l'Imam, per apprendere e recitare il corano, fa il pranzo assieme ad altri ragazzi. «... Questa volta non sono rientrato nell'Islam, non con l'idea politica della guerra santa, ma con l'idea della religione.»

L'analisi di questo caso mette in evidenza quali possono essere gli effetti pato-facilitatori della religione, quando viene sostituita al vuoto profondo esistenziale, particolarmente come scelta controreattiva ad una profonda frustrazione e umiliazione.

Il primo avvicinamento all'Islam è stato di tipo integralista, alla ricerca, di un ruolo definito, anche violento, per ristrutturare una personalità che si era sgretolata. Il ricovero coattivo ha agito come contro trauma psichico riportandolo ad una scelta più affine al suo IO. Da allora la religione, non più vissuta come una «setta»chiusa, integralista, ma aperta al Se Psichico e Sociale, è diventata pato-protettiva salvaguardandolo da eventuali futuri scompensi psichici.

I benefici che egli rileva sono: serenità, appartenenza, socializzazione, riduzione significativa degli stati di aggressività, migliore rapporto con le figure di attaccamento.

Il terzo caso clinico che viene descritto concerne un uomo del 1964. Nato in India, nel Punjab. Il padre è deceduto quando aveva otto anni. La madre è vivente. Quartogenito di una prole costituita da due fratelli e una sorella. Terminato il liceo, fu arruolato nell'esercito dove rimase per sei anni e mezzo. Partecipò attivamente alla guerra in Sri Lanka.

A causa del rischio di venire incarcerato per questioni politiche in India decise di emigrare in Svizzera. Arrivò nel 1993, come rifugiato politico. Dopo qualche anno ottenne il permesso di dimora. In India era sposato. In Svizzera arrivò da solo, lasciando la propria famiglia, la moglie e due figl. Divorziato nel 2004.

Dal lato diagnostico siamo nell'area depressiva con elementi trumatici legati ai gravi vissuti originanti dalle devastanti esperienze di guerra. Di area religiosa Induista. Tutte le mattine effettua l'adorazione induista per circa un'ora davanti a un tempietto che ha in casa "mi ha sempre aiutato per le ansie e la depressione».

La preghiera avviene sempre davanti a questo piccolo tempio con profumi e candele. Ha un libro da leggere.

"... si parla davanti a Dio, lui ascolta, io sento dentro fedeltà e fiducia che lui mi guarda e mi aiuta. Da quando sto male faccio questo ... prima non lo facevo ... faccio la preghiera ove Dio non mi abbondona perché ho paura che succeda qualcosa alla mia persona ... in India ci sono tanti posti ove esce acqua dolce e latte le persone bevono e stanno meglio ... allora Dio c'è ...».
I fattori pato-protettivi per lo sviluppo di una psicopatologia più grave ritrovati sono stati: riduzione dell'ansia e del senso di solitudine, riduzione dei pensieri negativi depressivi, forte senso di appartenenza, annullamento dei pensieri auto soppressivi.

Il quarto caso clinico illustra la storia di una donna del 1970. Di origini abruzzesi, è nata e cresciuta in Svizzera. Il padre viene definito simbolicamente come un padre padrone. La madre sarebbe stata sempre una donna sottomessa. Ha un fratello. Sposata, una prima volta, nel 1992 e divorziata nel 1995. Dal matrimonio nacque una figlia, affetta da un gravissimo morbo di Crohn, con fistoli anali. Sposata, una seconda volta nel 2013.

Dal lato diagnostico siamo nell'area della disfunzione vegetativa somatoforme con uno stato d'ansia di base e crisi di panico. La sintomatologia presentata apparteneva all'incapacità nel deglutire i solidi. Inoltre aveva una forte preoccupazione per la figlia che da molti anni si sottopone a cure specifiche senza un beneficio reale. Di fede Cristiana, ha trovato un sostegno particolarmente forte, nella chiesa evangelica. Ha iniziato a seguire la chiesa evangelica nel 2009.

I benefici evidenziati dell'appartenenza all'area evangelica sono stati: miglioramento degli stati ansiosi, riduzione significativa del sintomo con nuovamente capacità ad assumere i cibi solidi. Ha ripreso maggiore sicurezza in se stessa riuscendo a dare fiducia al genere maschile, autorizzandosi ad innamorarsi e a sposarsi. Riduzione significativa delle crisi timiche. Dal alto psicoterapico è stata impostata una terapia cognitivo comportamentale e sistemico relazionale individuale.

Nel momento in cui il sistema terapeutico è diventato permeabile alle credenze religiose e le ha integrate nel percorso di cura, vi è stata una chiara accelerazione del miglioramento clinico e del senso di appartenenza.

Il quinto caso clinico dissertato è relativo alla complessa storia di un uomo del 1982. Nato e cresciuto in Svizzera. Genitori cattolici non praticanti, viventi. Il padre è medico, affetto da un disturbo bipolare, che gli ha impedito negli ultimi anni di esercitare la professione. Il padre è stato ricoverato in clinica psichiatrica a diverse riprese, così come la moglie.

La madre, infermiera, depressa ed ansiosa. Un primo fratello con sindrome depressiva., anch'egli ricoverato una volta in ambiente psichiatrico. La sorella più piccola soffre di un grave disturbo del comportamento alimentare. Ricoverata in cliniche specifiche, con un lungo percorso comunitario. Egli ha portato a termine la maturità liceale, dopo avere ripetuto quattro volte l'ultimo anno.

Dal lato diagnostico siamo nell'area dell'ansia sociale con crisi di panico e uno stato depressivo. La sintomatologia che lo affligge di più è, però, quella dell'area del dolore.

«... Gli occhi mi fanno male tutto il giorno, non un dolore acuto ma persistente, occhi pesanti. Il naso lo soffio tutto il giorno, mi dà fastidio il naso chiuso ... Il collo mi fa molto male, da alcuni mesi mi crea problemi per il lavoro ... la schiena mi fa male tutto il giorno, già da quando mi sveglio, mi dà problemi al lavoro ... ma 
riesco a lavorare perché sono forte. Le braccia mi fanno male se tocco dal polso fino al braccio, anche le mani mi fanno male ... faccio fatica a fare dei movimenti ... il torace mi fa male dalla parte alta e poi scivola nella parte laterale fino alle anche. La coscia mi fa male la parte davanti della coscia, il dolore è diffuso ... il dolore si diffonde poi ai glutei, ai genitali, alla lingua, al cuoio capelluto, alle dita ed alla pianta dei piedi, alla gola, ai bronchi, ai polmoni, alla pleura ed al cuore ...».

Da alcuni anni si è interessato alla religione Buddista.

«sono diventato Buddista perché volevo vivere in una dimensione spirituale e avere qualcosa che mi sostenesse nei momenti difficili ... all'inizio ho avuto difficoltà, perché ci si rende conto che l'approccio Buddista è molto diverso dal nostro mondo veloce e turbolento ... il Buddismo ci porta a fermarci ... all'inizio è molta fatica perché avevo la paura e l'ansia che mi bloccavano ... adesso so che la paura posso combatterla con emozioni positive ...».

I benefici che egli ha sottolineato sono relativi alle pratiche meditative mattutine e vesperali. «... Mi possono far combattere e sostenere i dolori ...». Sente di aver rinforzato la capacità di tollerare il dolore. La soglia dolorifca si è alzata e la percezione del dolore è diventata meno sensibile.

Nel setting di psicoterapia la sensibilità verso l'area spirituale e la curiosità terapeutica (Cloninger et al., 1993) hanno permesso di includere la dimensione buddista nel percorso di terapia. Il terapeuta non dovrebbe mai dare per acquisita l'appartenenza religiosa.

Infatti in questo caso clinico emerge come la prima religione era quella cattolica cristiana. Solo l'interesse allargato e le domande specifiche sull'area della spiritualità hanno permesso di percorrere e comprendere da dove arrivava il miglioramento clinico.

Non erano solo i farmaci, non era solo la combinazione dei diversi approcci psicoterapeutici adottati negli anni, dalla terapia sistemico familiare a quella cognitiva comportamentale, alla mindfulness, al training autogeno, ma anche lo stimolo ad entrare in un percorso di Buddismo evolutivo.

L'ultimo caso clinico narrato coinvolge la storia di una donna di 71 anni, nata e cresciuta in Campania, regione posta nel sud dell'Italia. Emigrata in Svizzera Tedesca negli anni settanta.

Sposata una prima volta con un uomo maltrattante. Divorziata e risposata dopo qualche anno. Scoprì qualce anno fa che il secondo marito, contraddistinto da una forte personalità narcisistica, avrebbe sempre avuto una vita parallela con frequentazione di molte altre donne. Nei suoi confronti il marito era persecutorio e limitante delle sue libertà. Riuscì a separarsi alcuni anni orsono, decidendo di allontanarsi dalla Svizzera Tedesca e trasferirsi in Ticino.

Dal lato diagnostico emerge l'area depressiva all'interno di una storia personale caratterizzata da relazioni di- sturbate e fortemente inibitorie il suo sviluppo personale. È sempre stata di fede Cattolica Cristiana. Estremamente credente. Durante i periodi più complessi della sua vita ha sempre rinforzato l'appartenenza ai gruppi di preghiera incontrando un forte misticismo, a tratti sfociante in dimensioni, che dal lato psichiatrico tenderebbe a far propendere per l'insorgenza di un delirio religioso.

Integrando le pratiche religiose seguite all'interno del suo percorso di vita ed essendo sempre state pratiche pato protettive, anche se sfocianti in momenti simil deliranti, il terapeuta le ha fatte divenire parte attiva del setting. Così facendo ha rinforzato la fiducia in terapia, autorizzando la persona ad entrare in un ambiente sicuro, non aggressivo o squalificante.

I benefici evidenziati risiedono nel superamento delle crisi maggiori attraverso la preghiera e la contemplazione. Attraverso la preghiera e la contemplazione riesce a raggiungere una riduzione significativa dei propri stati d'ansia e di inquietudine, giungendo anche a superare fenomeni dispercettivi con ombre e voci che solitamente vive nei momenti di crisi importanti. È stato molto curativo l'aver affrontato i fenomeni dispercettivi nel setting terapeutico. L'apertura del setting a questi aspetti ha autorizzato il pensiero a defluire dal rischio di un incistamento deviante e delirante.

L'accoglienza non giudicante e non stigmatizzante ha sciolto il rischio della deriva psicotica e le pratiche religiose sono state riattribuite ad una particolarità propria della storia personale e hanno assunto carattere di autoterapia.

\section{Conclusioni}

Dai casi clinici descritti si evince come nella pratica Buddista e Induista la meditazione, il rapporto con la divinità, la forte connotazione della spiritualità, anche senza la componente religiosa attiva e praticante, può portare ad un rafforzamento del proprio IO. Nella religione Cristiana la pratica attiva ha evidenziato il rafforzamento del senso gruppale, la socializzazione e l'integrazione, attraverso anche le preghiere e gli incontri in comune. Altri punti significativi sono stati la migliore aderenza alle norme sociali, uno stato di contemplazione condivisa e la creazione di una forte rete di supporto.

La Spiritualità, dal suo canto, può avere un effetto protettivo sui life's stressors costruendo e rinforzando un senso di pace interiore capace di contenerli. Considerato l'analisi dei casi clinici e della letteratura, diventa significativo per ogni terapeuta raccogliere nell'anamnesi le caratteristiche della religiosità, della spiritualità, ma anche dell'ateismo.

Sarà importante sviluppare la capacità di parlare coi pazienti della spiritualità, della religione e delle credenze, comunicando senso di empatia e di rispetto. Sviluppare la capacità di supportare e rinforzare il coping spirituale e religioso, come la contemplazione, la meditazione, le preghiere, la partecipazione ad incontri coi gruppi di appartenenza, risulta essere fortemente terapeutico. 
La forza terapeutica passa attraverso il rinforzo dell'alleanza terapeutica che stimolerà il miglioramento del senso di benessere, con conseguente riduzione degli stati depressivi, ansiosi, del consumo di alcool e droghe, nonché una riduzione dei tassi di suicidio. La presenza di una strutturata correlazione positiva fra religione e senso di benessere può ripristinare il senso di tranquillità interiore e una maggiore stabilità. Rinforzando l'appartenenza religiosa o le pratiche spirituali/religiose il soggetto può sviluppare altre connessioni psicosociali che possono permettere di rinforzare il processo di cura e di miglioramento.

La relazione terapeutica può divenire sempre più personale rinforzando i fattori terapeutici non specifici formulati da Rogers: genuinità, empatia e un incondizionato sguardo positivo (Rogers, 1957).

In un periodo storico caratterizzato da forte senso di disillusione, da un'accentuazione della solitudine e da un senso di perdita della speranza, ciò rappresenta un rinforzo della relazione clinica e della sensibilità terapeutica e, finalmente, del prendersi cura del soggetto.

\section{Bibliografia}

American Psychiatric Association. (2000). Diagnostic and statistical manual of mental disorders (4th ed., text rev.). Washington, D.C.

Cecchin, G., Lane, G. \& Ray, W.A. (1997). Verità e Pregiudizi. Un approccio sistemico alla psicoterapia. Mailand: Raffaello Cortina.

Cloninger, C. R., Bayon, C. \& Svrakic, D. M. (1998). Measurement of temperament and character in mood disorders: a model of fundamental states as personality types. Journal of Affective Disorders, $51(1), 21-32$.

Cloninger, C. R., Svrakic, D. M. \& Przybeck, T.R. (1993). A Psychobiological Model of Temperament and Character. Archives of General Psychiatry, 50(12), 975-990.

Colucci, E. \& Martin, G. (2008). Religion and Spirituality Along the Suicidal Path. Suicide and Life-Threatening Behavior, 38(2), 229244.

Enstrom, J.E. (1989). Health Practices and Cancer Mortality among Active California Mormons. Journal of the National Cancer Institute, $81,1807-1814$

Gay, P. (1987). A Godless Jew: Freud, Atheism, and the Making of Psychoanalysis. New Haven: Yale University Press.

Goldbourt, U., Yaari, S. \& Medalie, J.H. (1993). Factors Predictive of Long-Term Coronary Heart Disease Mortality among 10,059 Male Israeli Civil Servants and Municipal Employees. A 23-Year Mortality Follow-up in the Israeli Ischemic Heart Disease Study. Cardiology, 82, 100-121.

Koenig, H. G., McCullough, M.E. \& Larson, D. B. (2001). Handbook of Religion and Health. Oxford: University Press.

Koenig, L. B., McGue, M., Krueger, R.F. \& Bouchard Jr., T. J. (2005). Genetic and Environmental Influences on Religiousness: Findings for Retrospective and Current Religiousness Ratings. Journal of Personality, 73(2),471-488.
Larson, D. B., Swyers, J.P. \& McCullough, M.E. (1997). Scientific Research on Spirituality and Health: A Consensus Report. Rockville/ MD: National Institute for Health Research.

Mattia, M., Barbetta, P. \& Pakman, M. (2015). Abstract 4 congresso mondiale WACP.

Mohr, S., Brandt, P. Y., Gillieron, C., Borras, L. \& Huguelet, P. (2006). Toward an Integration of Spirituality and Religiousness Into the Psychosocial Dimension of Schizophrenia. American Journal of Psychiatry, 163, 1952-1959.

Mohr, S. \& Huguelet, P. (2004). The Relationship between Schizophrenia and Religion and Its Implications for Care. Swiss Medical Weekly, 134, 369-376.

Muramoto, O. (2004). The role of the medial prefrontal cortex in human religious activity. Medical Hypotheses, 62(4), 479-485.

Neeleman, J. \& King, M.B. (1993). Psychiatrists' religious attitudes in relation to their clinical practice: A survey of 231 psychiatrists. Acta Psychiatrica Scandinavica, 88, 420-424.

Newberg, A., Alavi, A., Baime, M., Pourdehnad, M., Santanna, J. \& D'Aquili, E. (2001). The Measurement of Regional Cerebral Blood Flow during the Complex Cognitive Task of Meditation: A Preliminary SPECT Study. Psychiatry Research, 106(2), 113-122.

Newberg, A. \& Iversen, J. (2003). The Neural Basis of the Complex Mental Task of Meditation: Neurotransmitter and Neurochemical Considerations. Medical Hypotheses, 61(2), 282-291.

Newberg, A., Pourdehnad, M., Alavi, A. \& D’Aquili, E. G. (2003). Cerebral Blood Flow During Meditative Prayer: Preliminary Findings and Methodological Issues. Perceptual and Motor Skills, 97(2), 625-630.

Rogers, C.R. (1957). The Necessary and Sufficient Conditions of Therapeutic Personality Change. Journal of Consulting Psychology, 21(2), 95-103.

Yangarber-Hicks, N. (2004). Religious Coping Styles and Recovery from Serious Mental Illness. Journal of Psychology and Theology, $32,305-317$.

\section{L'autore}

Michele Mattia, FMH Psichiatria e Psicoterapia, Presidente Associazione della Svizzera Italiana per l'Ansia, la Depressione e il Disturbo Ossessivo-Compulsivo (Asi-Adoc), Docente presso L’Università di Varese, dipartimento di Psichiatria; Didatta presso il Centro Milanese Terapia della Famiglia.

\section{Contattateci}

Viale San Salvatore 7 6902 Lugano-Paradiso Tel +41916471417

E-Mail: studiomattia@michelemattia.ch www. michelemattia.ch 